\title{
Perceptions and Reproductive Health Seeking Behaviors of University of Ghana Female Students
}

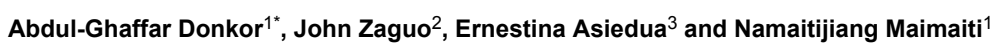 \\ ${ }^{1}$ Department of Public Health, Ankara Yildirim Beyazit University, Turkey \\ ${ }^{2}$ School of Nursing and Midwifery, University of Ghana, Legon, Ghana \\ ${ }^{3}$ Department of Maternal and Child Health, School of Nursing and Midwifery, University of Ghana, Legon, Ghana
}

*Corresponding author: Abdul-Ghaffar Donkor, Public Health Department, Faculty of Medicine, Ankara Yildirim Beyazit University, Bilkent Caddesi, Ankara, Turkey, Tel: +00905439796989; E-mail: abdulghaffardonkor@yahoo.com

Received date: May 18, 2018; Accepted date: May 26, 2018; Published date: June 06, 2018

Copyright: ( 2018 Donkor AG, et al. This is an open-access article distributed under the terms of the Creative Commons Attribution License, which permits unrestricted use, distribution, and reproduction in any medium, provided the original author and source are credited.

\begin{abstract}
Objective: The aims of the study were to explore university female students' perception about reproductive health problems, their reproductive health seeking behaviors and how reproductive health problems could be managed from their perspective.
\end{abstract}

Methodology: A cross-sectional survey was conducted using self-administered questionnaires among university female students in University of Ghana. These were students who were enrolled into any undergraduate program in the university. Data was collected using self-administered standardized questionnaire on perceptions and reproductive health problems among 50 students from university of Ghana female students who agreed to participate in the study using stratified random sampling technique.

Result: The mean age of the respondents was 25 years. Out of the 50 participants, $70 \%$ could define reproductive health correctly whilst $30 \%$ could not give accurate definition of reproductive health. $84 \%$ of respondents were able to mention at least one of such reproductive problems. Twenty-three (23) respondents which represent $54.8 \%$ of the 42 respondents who had heard about some reproductive health problems said reproductive health problems can be prevented through sex education, 7 respondents representing $16.7 \%$ mentioned abstinence as a way of preventing reproductive health problems, 3 people mention family planning and this constitute $7.1 \%$ of the 42 respondents. In addition, 4 respondents which constitute $9.5 \%$ mentioned medical interventions such as seeking for a safe abortion at well-equipped institutions with qualified personnel as a way of preventing reproductive health problems. Majority of the participants, 32(64\%) were aware of interventions aimed at addressing reproductive health problems whilst 18 participants representing $36 \%$ were not aware.

Conclusion: Findings from this study indicate great awareness of reproductive health problems and sex education among the respondents. $100 \%$ of the respondents who have ever had an STI sought treatment from either the hospital or the pharmacy.

Keywords: Perceptions; Reproductive health seeking behaviors; University of Ghana; Female students

\section{Introduction}

According to Population Reference Bureau 2012, more than a quarter of the world's population is between the ages of 10-24 years of which 1.8 billion young people live in developing countries. Ghana's population in 2010 was estimated at 24.6 million. Of this, young people age $10-24$ years constitute $31.8 \%$ of the population.

The major reproductive health problems among women in Ghana include but not limited to the following; Increase in the incidence of sexually transmitted infections, unintended Pregnancies and Unsafe abortions.

Unsafe abortion is a procedure for terminating an unintended pregnancy either by a person lacking the necessary skills or in an environment lacking the minimal medical standard or both [1]. During 2010-2014, an estimated 56 million induced abortions occurred each year worldwide [2]. Globally, $25 \%$ of pregnancies ended in abortion in 2010-2014. In developed countries, the proportion declined from $39 \%$ to $28 \%$ between 1990-1994 and 2010-2014, whereas it increased from $21 \%$ to $24 \%$ in developing countries during 2010-2014, an estimated 8.3 million induced abortions occurred each year in Africa [2].

Unintended pregnancy is one that occurs at a time that a woman does not want to get pregnant sooner than she would have desired or when she desired or no more want children [3]. It is estimated that 213 million pregnancies occurred worldwide in 2012, up slightly from 211 million in 2008. The global pregnancy rate decreased only slightly from 2008 to 2012, after declining substantially between 1995 and 2008. 85 million pregnancies representing $40 \%$ of all pregnancies were unintended in 2012. Of these, 38\% resulted in unplanned births. With the 213 million pregnancies that occurred worldwide in 2012.

190 million (89\%) of all pregnancies occurred in the developing world. More than half of all pregnancies (56\%) took place in Asia, 25\% 
in Africa, and 8\% in Latin America and the Caribbean, 7\% in Europe and $3 \%$ in North America and less than $1 \%$ in Oceania.

Sexually Transmitted Infections are those infections that are usually transmitted through sexual intercourse (vaginal, oral and anal) [1]. More than 30 different bacteria, viruses and parasites are known to be transmitted through sexual contact. Eight of these pathogens are linked to the greatest incidence of sexually transmitted disease. Of these 8 infections, 4 are currently curable: syphilis, gonorrhea, chlamydia and trichomoniasis. The other 4 are viral infections and are incurable: hepatitis B, herpes simplex virus (HSV or herpes), HIV, and human papillomavirus (HPV) [1]. Globally, more than 1 million STIs are acquired every day with annual estimates at 357 million new infections with one of four STIs: chlamydia (131 million), gonorrhea (78 million), syphilis (5.6 million) and trichomoniasis (143 million) [1].

Despite WHO efforts through the sustainable development goals, other government and non-governmental organizations to improve reproductive health among women of various age groups, reproductive health problems-unintended pregnancies, unsafe abortions and sexually transmitted infections among women are still on the rise. This study therefore, was conducted to assess the perceptions and reproductive health seeking behaviors of University of Ghana female students.

\section{Aims}

The aims of the study were to explore university females' perception about reproductive health problems, their reproductive health seeking behaviors and how reproductive health problems can be managed from their perspective.

\section{Methodology}

\section{Study design and data collection}

A cross-sectional survey was conducted using self-administered questionnaires among female students in University of Ghana. These were students who were enrolled into any undergraduate program in the university. Students were informed about the study and encouraged to participate. Those who agreed to participate were given questionnaire to fill. The questionnaires were distributed to the students at the end of their scheduled class sessions and at their hall of residence. The questionnaires took an average of twenty minutes to be completed. The completed questionnaires were retrieved immediately after completing them.

\section{Sample size and sample selection}

There were 50 respondents in the study. Stratified random sampling technique was used to recruit participants. Questionnaires were administered randomly to female students at their halls of residence and lecture halls. The respondents ranged from level 100 to level 400 in various courses in the university.

\section{Study location}

The study was conducted at the University of Ghana, Legon main campus. It is the oldest and largest public university in Ghana. PostGraduate degree programs, Bachelors' Degrees, Sub-Degrees are being run in the university. The university has both local and international students currently enrolled in the University. The University is centered around four (4) colleges, six (6) institutes, nineteen (19) schools, five (5) centers of research and learning and ninety-five (95) departments.

\section{Questionnaire}

A self-administered structured questionnaire was used as the main tool for the data collection. The questionnaire was designed by the researchers themselves guided by the objectives of the study. The questionnaires were given to respondents to answer and collected with the help of female friends. The questionnaire contained both closedended questions and few open-ended questions. The questionnaire was divided into four sections; section collected demographic information of the respondents. Section $\mathrm{B}$ elicited the perception about reproductive health problems. Section $C$ contained questions that assessed the participants' health seeking behaviors and section $\mathrm{D}$ also contained questions to ascertain the ways of addressing reproductive health problems.

\section{Ethical considerations}

The study proposal was approved by the Ethic committee of University of Ghana Nursing School. Prior to data collection, all study participants were given information on the study and assured that all data is confidential and will only be analyzed as aggregates. All respondents had informed consent form before participation.

\section{Results}

The data from the questionnaire was processed and analyzed by computer using the Statistical Package for Social Sciences (SPSS) version 16.0. The results were presented using descriptive frequencies such as percentages and means.

\section{Participant's profile}

The data collected indicates a young population with a majority (74\%) of the respondents being between the ages of 21-24 years of age and an average age (mean age) of 25 years. Majority (48\%) of the respondents come from villages, followed by those from towns (36\%) and least from cities (16\%). Majority of the participants are also Christians (84\%) with a few being.

Muslims (16\%) In the study, it is realized that a greater proportion of the participants are in level 400 with a fraction of students from level 300,100 and 200 respectively. Sixty-four percent (64\%) of the respondents were single whiles $36 \%$ were in a relationship at the time of data collection. Meanwhile, there is no one who is married, divorced, separated or widowed. Respondents from the college of health sciences were $22(44 \%)$ and the college of humanities to was 22 (44\%). The college with the least number of participants in the study was the college of basic and applied sciences, 6 (12\%). In terms of residence, there were more participants from the traditional halls as compared with the hostels.

\section{Outcome of study}

\section{Perception about reproductive health problems}

Out of the 50 respondents in the study, 35 respondents (70\%) could define reproductive health correctly whilst fifteen (15) respondents which constitute $30 \%$ could not give accurate definition of reproductive health. $84 \%$ of respondents were able to mention at least 
one of such reproductive problems as sexually transmitted infections, teenage pregnancies, unsafe abortions and other reproductive health problems such as uterine fibroids. Also, 42 respondents of the 50 participants said they have heard about some reproductive health problems that bedevil women, 13 mentioned the media as their source of information and this constitute $31 \%$ of the valid percentage, 4 respondents representing $9.5 \%$ mentioned family and friends, 6 respondents representing $14.3 \%$ mentioned health professionals, 17 respondents which constitute $40.5 \%$ mentioned more than one of the above mentioned sources of information whilst 2 respondents representing $4.8 \%$ of the respondents mentioned other sources of information such as schools and the internet as their source of information on reproductive health problems. In addition, Twentythree (23) respondents which represent $54.8 \%$ of the 42 respondents who had heard about some reproductive health problems said reproductive health problems can be prevented through sex education, 7 respondents representing $16.7 \%$ mentioned abstinence as a way of preventing reproductive health problems, 3 people mention family planning (contraceptive use) and this constitute $7.1 \%$ of the 42 respondents. In addition, 4 respondents which constitute $9.5 \%$ mentioned medical interventions such as seeking for a safe abortion at well-equipped institutions with qualified personnel as a way of preventing reproductive health problems. Moreover, 5 people representing $11.9 \%$ mention more than one of the above named interventions. Moreso with the 50 respondents, 42 (84\%) of them said they had never had STI representing the highest percentage whilst 8 respondents (16\%) said they had ever contracted an STI before representing the least percentage. Furthermore, Out of the 50 respondents, majority of them (92\%) was able to define unintended pregnancy correctly while a small percentage $(8 \%)$ of the respondents could not define an unintended pregnancy (Figure 1).

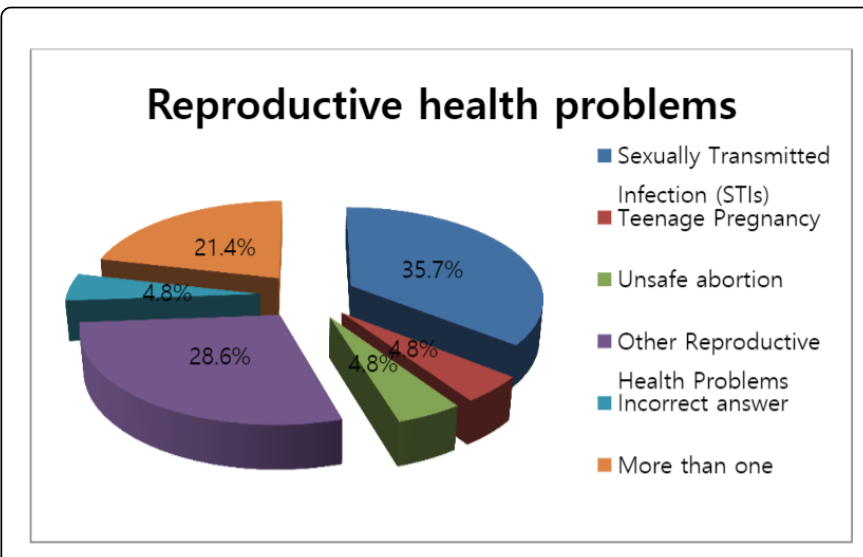

Figure 1: shows the percentages of those of who heard of some reproductive health problems.

\section{Reproductive health seeking behaviors of respondents}

It shows that all the $100 \%$ participants who had ever contracted an STI sought treatment for their condition indicating a positive step towards resolving their ailments. Majority of the participants (62.5\%) who ever had an STI sought treatment from the hospital and 37.5\% sought treatment from the pharmacist. Also, Seventy-four percent (74\%) of the respondents representing the majority said they will maintain unintended pregnancy while $26 \%$ representing the smallest percentage said they will terminate the pregnancy. $30 \%$ indicated they will terminate the pregnancy in a hospital. $2 \%$ indicated she will terminate the pregnancy in a pharmacy whilst another $2 \%$ said she will terminate the pregnancy from the herbalist. This indicates that majority of the respondents will terminate unintended pregnancy in a hospital. Twenty-eight (28\%) of the respondents who will like to terminate the pregnancy mentioned safety as the reason for their place of choice whilst $6 \%$ mentioned other reasons such as cost of treatment and the ability of the care provider to maintain their dignity and confidentiality as their reasons that inform their decision to seek treatment from their places of choice.

\section{Ways to manage reproductive health problems}

Majority of the participants, 32(64\%) were aware of interventions aimed at addressing reproductive health problems whilst 18 participants representing $36 \%$ were not aware. Table 1 shows the views of respondents on ways of addressing reproductive health problems. 12 $(37.5 \%)$ of the respondents who said they were aware of some interventions to manage reproductive health problems think sex education is the most effective strategy, 5 (15.6\%) respondents were of the view that family planning can be used to address reproductive health problems, $3.1 \%$ of respondents think people should abstain from sexual intercourse till marriage to address the reproductive health menace. Also, 4 (12.5\%) respondents believe that medical interventions such as safe abortion practices can be used to prevent people from carrying out unsafe abortions whilst 8 (25\%) respondents think more than one of these interventions mentioned above should be used to reduce the menace of reproductive health problems. Meanwhile, $2(6.2 \%)$ respondents who answered yes to the question of whether they are aware of any interventions for addressing reproductive health problems mention other interventions such as the establishment of adolescent friendly facilities, safe sex practices such as the use of condoms during sex as some of the potent strategies to effectively and efficiently manage the rise in reproductive health problems. Of the 50 respondents, 21 respondents (42\%) indicated that all the institutions listed (families, schools, religious bodies and health institutions) should be involved in managing reproductive health problems. 23 respondents $(46 \%)$ indicated more than one of the institutions listed should be involved. 3 respondents (6\%) indicated that only health institutions should be involved in addressing reproductive health problems and one respondent $(2 \%)$ each indicate that only the families, schools and religious bodies should be involved in addressing reproductive health problems.

\begin{tabular}{|l|l|l|}
\hline $\begin{array}{l}\text { Interventions for reproductive health } \\
\text { problems }\end{array}$ & $\begin{array}{l}\text { Frequency } \\
\text { (N) }\end{array}$ & $\begin{array}{l}\text { Valid Percent } \\
(\%)\end{array}$ \\
\hline Sex education & 12 & 37.5 \\
\hline Family planning & 5 & 15.6 \\
\hline Abstinence & 1 & 3.1 \\
\hline Medical Intervention & 4 & 12.5 \\
\hline Others & 2 & 6.2 \\
\hline More than one & 8 & 25 \\
\hline Total & 32 & 100 \\
\hline
\end{tabular}

Table 1: A Frequency table showing the views of participants on ways to prevent reproductive health problems. 


\section{Discussion}

From the study, it is evident that many of the respondents have knowledge on reproductive health. As much as $70 \%$ of the respondents could define reproductive health correctly. $84 \%$ of them being able to mention at least one of such problems as sexually transmitted infections, teenage pregnancies, unsafe abortions and other reproductive health problems such as uterine fibroids. Also, $34 \%$ of participants got the information from at least one of these sources; media, family and friends as well as health professionals indicating that people have become more conscious of matters relating to their reproductive health. These findings agree with those in earlier studies by Awusabo-Asare et al. The study findings show that participants have positive attitude in terms of seeking treatment for their reproductive health problems. This is evident in the response of the participants as $100 \%$ of the respondents who have ever had an STI sought treatment from either the hospital or the pharmacy. The participants mentioned safety and cost of the treatment regimen as well as ease of access to services as some of the reasons why they sought treatment from the hospital and the pharmacy. These findings are in contrast with earlier research that highlighted the point that treatment seeking for reproductive health problems is low among women in India. Another study conducted by Shagun et al. on treatment seeking for symptoms of reproductive tract infections among young women in India.

From the study, majority of the participants suggested sex education. This shows the level to which information concerning reproductive health problems is lacking as participants suggested.

Schools, family, religious bodies and health institutions should be actively involved in addressing reproductive health problems. This study supports previous related studies on the topic. A study by Biddlecom et al. on the topic Adolescents views of the preferences for sexual and reproductive health services in Burkina Faso, Ghana, Malawi and Uganda $[4,5]$.

The study explores the perceptions and reproductive health seeking behaviors of female students in University of Ghana. The study did not limit itself to only one faculty but all faculties in the university [6-10]. There was no age restriction either. Any female undergraduate student could participate. The major limitation of this study was the fact that the study was conducted using a sample in University of Ghana so may not necessarily represent the overall female students in the University.

\section{Conclusion}

This study was quantitative study with 50 respondents. It sought to assess the perceptions and reproductive health seeking behaviors of University of Ghana (UG) female students and the ways to address these reproductive health problems [11-12]. Findings from this study indicate great awareness of reproductive health problems and sex education among the respondents. $70 \%$ of the respondents could define reproductive health correctly whilst $30 \%$ could not give accurate definition of reproductive health. $84 \%$ of them being able to mention at least one of such problems as sexually transmitted infections, teenage pregnancies, unsafe abortions and other reproductive health problems such as uterine fibroids. $100 \%$ of the respondents who have ever had an STI sought treatment from either the hospital or the pharmacy. The participants mentioned safety and cost of the treatment regimen as well as ease of access to services as some of the reasons why they sought treatment from the hospital and the pharmacy.

\section{Recommendations}

Firstly, more education should be provided on reproductive health issues. Also, Evidence-based sex education must be a major strategy and should be integrated into the syllabuses and curricula at the basic level to imbue adolescents and young adults with knowledge at an early stage in their development which can positively influence their behavior choices. In addition, government and other stakeholders in the health sector must provide user friendly resources which are readily available to adolescents and young adults for early detection of reproductive health problems and in other to provide early solutions to them. Finally, it is important to educate adolescents about safe sex and contraceptives.

\section{Acknowledgement}

The authors would like to thank all students who participated in this study.

\section{References}

1. World Health Organization (2015) Global Strategy for the Prevention and Control of Sexually Transmitted Infections, 2006-2015. WHO: Geneva.

2. Guttmacher Institute (2016) Induced Abortion Worldwide. Global incidence and trends.

3. Singh S, Wulf D, Hussain R, Bankole A, Sedgh G (2009) Guttmacher Institute and United.

4. Abroso A (2013) Health literacy and Sexually Transmitted Infections (STI'S) among University of Ghana female students. University of Ghana: Legon.

5. Adanu RM, Seffah J, Anarfi JK, Lince N, Blanchard K (2012) Sexual and reproductive health in Accra. Ghana Medical Journal 46: 58-65.

6. Easa M, Roghaiyeh N, Masoumeh S (2015) Iranian Azeri Women's perceptions of Unintended Pregnancy.

7. Family Plannin (2012) The London Summit on family planning.

8. Iqbal S, Elisabeth A (2010) Unsafe Abortions in 2008: Global and Regional Levels and Trends. Reproductive Health Matters 18: 90-101.

9. Janice H (2011) Sexually Transmitted Infections. Pregnancy and Intimate Partner violence 32: 23-38.

10. Muzny CA, Harbison HS, Pembleton ES, Austin EL (2013) Sexual behaviors, perception of sexually transmitted infection risk, and practice of safe sex among southern African American women who have sex with women. Sex Transm Dis 40: 395-400.

11. Prusty RK, Unisa S (2013) Reproductive Tract Infections and Treatment Seeking Behavior among Married Adolescent Women 15-19 Years in India. Int J MCH AIDS 2: 103-110.

12. Nations Population fund (1967). University of Medical Sciences. 